\title{
STABILITY ANALYSIS OF A RATIO-DEPENDENT PREDATOR-PREY SYSTEM WITH DIFFUSION AND STAGE STRUCTURE
}

\author{
XINYU SONG, ZHIHAO GE, AND JINGANG WU
}

Received 16 November 2004; Revised 25 January 2006; Accepted 16 February 2006

A two-species predator-prey system with diffusion term and stage structure is discussed, local stability of the system is studied using linearization method, and global stability of the system is investigated by strong upper and lower solutions. The asymptotic behavior of solutions and the negative effect of stage structure on the permanence of populations are given.

Copyright @ 2006 Hindawi Publishing Corporation. All rights reserved.

\section{Introduction}

Predator-prey models have been studied by many authors (see $[6,21]$ ), but the stage structure of species has been ignored in the existing literature. In the natural world, however, there are many species whose individual members have a life history that take them through two stages: immature and mature (see [1-3, 7-9, 18-20]). In particular, we have in mind mammalian populations and some amphibious animals, which exhibit these two stages. In these models, the age to maturity is represented by a time delay, leading to a system of retarded functional differential equations. For general models one can see [11].

Specifically, the standard Lotka-Volterra type models, on which nearly all existing theories are built, assume that the per capita rate predation depends on the prey numbers only. An alternative assumption is that, as the numbers of predators change slowly (relative to prey change), there is often competition among the predators and the per capita rate of predation depends on the numbers of both preys and predators, most likely and simply on their ratio. A ratio-dependent predator-prey model has been investigated by $[10]$.

Recently, a model of ratio-dependent two species predator-prey with stage structure was derived in [19]. The model takes the form

$$
\begin{aligned}
& \frac{d X_{1}(t)}{d t}=\alpha X_{2}(t)-\gamma X_{1}(t)-\alpha e^{-\gamma \tau} X_{2}(t-\tau), \\
& \frac{d X_{2}(t)}{d t}=\alpha e^{-\gamma \tau} X_{2}(t-\tau)-\beta X_{2}^{2}(t)-\frac{c X_{2}(t) Y(t)}{X_{2}(t)+m Y(t)},
\end{aligned}
$$

Hindawi Publishing Corporation

International Journal of Mathematics and Mathematical Sciences

Volume 2006, Article ID 13948, Pages 1-20

DOI 10.1155/IJMMS/2006/13948 


$$
\begin{aligned}
& \frac{d Y(t)}{d t}=Y(t)\left(-d+\frac{f X_{2}(t)}{X_{2}(t)+m Y(t)}\right), \\
& x_{1}(0)>0, \quad y(0)>0, \quad x_{2}(t)=\varphi(t) \geq 0, \quad-\tau \leq t \leq 0,
\end{aligned}
$$

where $X_{1}(t), X_{2}(t)$ represent, respectively, the immature and mature prey populations densities; $Y(t)$ represents the density of predator population; $f>0$ is the transformation coefficient of mature predator population; $\alpha e^{-\gamma \tau} X_{2}(t-\tau)$ represents the immatures who were born at time $t-\tau$ and survive at time $t$ (with the immature death rate $\gamma$ ), and $\tau$ represents the transformation of immatures to matures; $\alpha>0$ is the birth rate of the immature prey population; $\gamma>0$ is the death rate of the immature prey population; and $\beta>0$ represents the mature death and overcrowding rate. The model is derived under the following assumptions.

(H1) The birth rate of the immature prey population is proportional to the existing mature population with a proportionality constant $\alpha>0$; the death rate of the immature prey population is proportional to the existing immature population with a proportionality constant $\gamma>0$; we assume for the mature population that the death rate is of a logistic nature.

(H2) In the absence of prey spaces, the population of the predator decreased, and $d>0$, $f>0, m>0$.

Note that the first equation of system (1.1) can be rewritten to

$$
X_{1}(t)=\int_{t-\tau}^{t} \alpha e^{-\gamma(t-s)} X_{2}(s) d s,
$$

so we have

$$
X_{1}(0)=\int_{-\tau}^{0} \alpha e^{\gamma s} X_{2}(s) d s .
$$

This suggests that if we know the properties of $X_{2}(t)$, then the properties of $X_{1}(t)$ can be obtained from $X_{2}(t)$ and $Y(t)$. Therefore, in the following we need only to consider the following model:

$$
\begin{gathered}
\frac{d X_{2}(t)}{d t}=\alpha e^{-\gamma \tau} X_{2}(t-\tau)-\beta X_{2}^{2}(t)-\frac{c X_{2}(t) Y(t)}{X_{2}(t)+m Y(t)}, \\
\frac{d Y(t)}{d t}=Y(t)\left(-d+\frac{f X_{2}(t)}{X_{2}(t)+m Y(t)}\right), \\
x_{1}(0)>0, \quad y(0)>0, \quad x_{2}(t)=\varphi(t) \geq 0, \quad-\tau \leq t \leq 0 .
\end{gathered}
$$

In [19], the effect of delay on the populations and the global asymptotic attractivity of the system (1.4) were considered, for detailed results we refer to [19]. However, the diffusion of the species which is in addition to the species' natural tendency to diffuse to areas of smaller population concentration is not considered. For the details of diffusion in different areas, we can see $[4,12-17,22]$. In this paper, we study the system (1.1) with 
diffusion terms, taking into account the diffusion of the species in different areas. The role of diffusion in the following system of nonlinear pdes with diffusion terms and stage structure will be studied:

$$
\begin{gathered}
\frac{\partial u_{1}}{\partial t}-D_{1} \Delta u_{1}=\alpha u_{2}(x, t)-\gamma u_{1}(x, t)-\alpha e^{-\gamma \tau} u_{2}(x, t-\tau), \\
\frac{\partial u_{2}}{\partial t}-D_{1} \Delta u_{2}=\alpha e^{-\gamma \tau} u_{2}(x, t-\tau)-\beta u_{2}^{2}(x, t)-\frac{c u_{2}(x, t) v(x, t)}{u_{2}(x, t)+m v(x, t)}, \\
\frac{\partial v}{\partial t}-D_{2} \Delta v=v(x, t)\left(-d+\frac{f u_{2}(x, t)}{u_{2}(x, t)+m v(x, t)}\right), \quad x \in \Omega, t>0, \\
\frac{\partial u_{1}}{\partial n}=\frac{\partial u_{2}}{\partial n}=\frac{\partial v}{\partial n}=0, \quad x \in \partial \Omega, t>0, \quad v(x, 0)=\varphi_{3}(x, 0), \quad x \in \bar{\Omega}, t \in[-\tau, 0],
\end{gathered}
$$

where $\partial / \partial n$ is differentiation in the direction of the outward unit normal to the boundary $\partial \Omega$, we assume $\Omega \subset \mathbb{R}^{N}$ is open, bounded and $\partial \Omega$ is smooth. The diffusion coefficients $D_{1}$, $D_{2}$, and $D_{3}$ are positive. The homogeneous Neumann boundary condition indicates that the predator-prey system is self-contained with zero population flux across the boundary. The initial functions $\varphi_{1}(x, t), \varphi_{2}(x, t)$, and $\varphi_{3}(x, t)$ are Hölder continuous, and satisfy the compatible condition

$$
\frac{\partial \varphi_{i}}{\partial n}=0 \quad \text { on } \partial \Omega, i=1,2,3
$$

Denote $u_{2}(x, t)$ and $v(x, t)$ as $u_{1}(x, t)$ and $u_{2}(x, t)$, respectively, so we get the following subsystem of the system (1.5):

$$
\begin{gathered}
\frac{\partial u_{1}}{\partial t}-D_{1} \Delta u_{1}=\alpha e^{-\gamma \tau} u_{1}(x, t-\tau)-\beta u_{1}^{2}(x, t)-\frac{c u_{1}(x, t) u_{2}(x, t)}{u_{1}(x, t)+m u_{2}(x, t)}, \\
\frac{\partial u_{2}}{\partial t}-D_{2} \Delta u_{2}=u_{2}(x, t)\left(-d+\frac{f u_{1}(x, t)}{u_{1}(x, t)+m u_{2}(x, t)}\right), \quad x \in \Omega, t>0, \\
\frac{\partial u_{1}}{\partial n}=0, \quad \frac{\partial u_{2}}{\partial n}=0, \quad x \in \partial \Omega, t>0, \\
u_{1}(x, t)=\varphi_{1}(x, t), \quad u_{2}(x, t)=\varphi_{2}(x, 0), \quad x \in \bar{\Omega}, t \in[-\tau, 0] .
\end{gathered}
$$

Note that the quantities $u_{2}(x, t)$ and $v(x, t)$ of the system (1.5) are independent of the quantity $u_{1}(x, t)$, so we may only consider the subsystem (1.7) to be easy to get the properties of the system (1.5).

Before proceeding further, let us nondimensionalize the system (1.7) with the following scaling: $U_{1}=\beta u_{1}, U_{2}=m \beta u_{2}, T=t$, by rewriting $U_{1}, U_{2}, T$ to $u_{1}, u_{2}$, $t$, respectively. 
We obtain the following nondimensionless system:

$$
\begin{gathered}
\frac{\partial u_{1}}{\partial t}-D_{1} \Delta u_{1}=a u_{1}(x, t-\tau)-u_{1}^{2}(x, t)-\frac{b u_{1}(x, t) u_{2}(x, t)}{u_{1}(x, t)+u_{2}(x, t)}, \\
\frac{\partial u_{2}}{\partial t}-D_{2} \Delta u_{2}=u_{2}(x, t)\left(-d+\frac{f u_{1}(x, t)}{u_{1}(x, t)+u_{2}(x, t)}\right), \quad x \in \Omega, t>0, \\
\frac{\partial u_{1}}{\partial n}=0, \quad \frac{\partial u_{2}}{\partial n}=0, \quad x \in \partial \Omega, t>0, \\
u_{1}(x, t)=\varphi_{1}(x, t), \quad u_{2}(x, t)=\varphi_{2}(x, 0), \quad x \in \bar{\Omega}, t \in[-\tau, 0],
\end{gathered}
$$

where $a=\alpha e^{-\gamma \tau}, b=c / m$.

The remaining part of this paper is organized as follows. The existence and uniqueness of the solutions of system (1.8) will be proved in Section 2. In Section 3, we obtain conditions for local asymptotic stability of the nonnegative equilibria of system (1.8). In Section 4, we analyze the global asymptotic stability and obtain conditions for global asymptotic stability of the nonnegative equilibria of system (1.8).

\section{Existence and uniqueness of the solutions}

In order to solve the problem and prove theorems, we devote to some preliminaries. We rewrite system (1.8) to

$$
\begin{gathered}
\frac{\partial u_{1}}{\partial t}-D_{1} \Delta u_{1}=F_{1}\left(u_{1}(x, t), u_{2}(x, t), u_{1}(x, t-\tau)\right), \\
\frac{\partial u_{2}}{\partial t}-D_{2} \Delta u_{2}=F_{2}\left(u_{1}(x, t), u_{2}(x, t)\right), \quad x \in \Omega, t>0, \\
\frac{\partial u_{1}}{\partial n}=0, \quad \frac{\partial u_{2}}{\partial n}=0, \quad x \in \partial \Omega, t>0, \\
u_{1}(x, t)=\varphi_{1}(x, t), \quad u_{2}(x, 0)=\varphi_{2}(x, 0), \quad x \in \bar{\Omega}, t \in[-\tau, 0],
\end{gathered}
$$

where $F_{1}\left(u_{1}(x, t), u_{2}(x, t), u_{1}(x, t-\tau)\right)=a u_{1}(x, t-\tau)-u_{1}^{2}(x, t)-b u_{1}(x, t) u_{2}(x, t) /$ $\left(u_{1}(x, t)+u_{2}(x, t)\right)$, and $F_{2}\left(u_{1}(x, t), u_{2}(x, t)\right)=u_{2}(x, t)\left(-d+f u_{1}(x, t) /\left(u_{1}(x, t)+u_{2}(x, t)\right)\right)$.

Definition 2.1. Suppose $\varphi_{1}(x, t), \varphi_{2}(x, t), \psi(x, t)$ be Hölder continuous, call $\left(\tilde{u}_{1}, \tilde{u}_{2}\right)$, $\left(\hat{u}_{1}, \hat{u}_{2}\right)$ to be a pair of strong upper and lower solutions, if $\tilde{u}_{1}, \hat{u}_{1}, \tilde{u}_{2}$, and $\hat{u}_{2} \in C(\bar{\Omega} \times$ $[0,+\infty)) \cap C^{2,1}(\Omega \times[0,+\infty))$ such that $\hat{u}_{1} \leq \tilde{u}_{1}, \hat{u}_{2} \leq \tilde{u}_{2}$, and

$$
\begin{gathered}
\frac{\partial \tilde{u}_{1}}{\partial t}-D_{1} \Delta \tilde{u}_{1} \geq a \tilde{u}_{1}(x, t-\tau)-\tilde{u}_{1}^{2}(x, t)-\frac{b \tilde{u}_{1}(x, t) \hat{u}_{2}(x, t)}{\tilde{u}_{1}(x, t)+\hat{u}_{2}(x, t)}, \\
\frac{\partial \hat{u}_{1}}{\partial t}-D_{1} \Delta \hat{u}_{1} \leq a \hat{u}_{1}(x, t-\tau)-\hat{u}_{1}^{2}(x, t)-\frac{b \hat{u}_{1}(x, t) \tilde{u}_{2}(x, t)}{\hat{u}_{1}(x, t)+\tilde{u}_{2}(x, t)}, \\
\frac{\partial \tilde{u}_{2}}{\partial t}-D_{2} \Delta \tilde{u}_{2} \geq-d \tilde{u}_{2}(x, t)+\frac{f \tilde{u}_{1}(x, t) \tilde{u}_{2}(x, t)}{\tilde{u}_{1}(x, t)+\tilde{u}_{2}(x, t)}
\end{gathered}
$$




$$
\begin{gathered}
\frac{\partial \hat{u}_{2}}{\partial t}-D_{2} \Delta \hat{u}_{2} \leq-d \hat{u}_{2}(x, t)+\frac{f \hat{u}_{1}(x, t) \hat{u}_{2}(x, t)}{\hat{u}_{1}(x, t)+\hat{u}_{2}(x, t)}, \quad x \in \Omega, t>0, \\
\frac{\partial \hat{u}_{1}}{\partial n} \leq 0 \leq \frac{\partial \tilde{u}_{1}}{\partial n}, \quad \frac{\partial \hat{u}_{2}}{\partial n} \leq 0 \leq \frac{\partial \tilde{u}_{2}}{\partial n}, \quad(x, t) \in \partial \Omega \times[0,+\infty), \\
\hat{u}_{1}(x, t) \leq \varphi_{1}(x, t) \leq \tilde{u}_{1}(x, t), \quad(x, t) \in \bar{\Omega} \times[-\tau, 0], \\
\hat{u}_{2}(x, 0) \leq \varphi_{2}(x, 0) \leq \tilde{u}_{2}(x, 0), \quad x \in \bar{\Omega} .
\end{gathered}
$$

Similar to Definition 2.1, the definition of a pair of strong upper and lower solutions of the elliptic system corresponding to system (2.1) is easy to be given.

Lemma $2.2[14]$. Suppose that $u_{i}(x, t) \in C(\bar{\Omega} \times[0, T]) \cap C^{2,1}(\Omega \times[0, T])$ satisfy

$$
\begin{gathered}
\frac{\partial u_{i}}{\partial t}-D_{i} \Delta u_{i} \geq \sum_{j=1}^{2} b_{i j} u_{j}(x, t)+\sum_{j=1}^{2} c_{i j} u_{j}\left(x, t-\tau_{i}\right), \quad(x, t) \in \Omega \times[0, T], \\
\frac{\partial u_{i}}{\partial n} \geq 0,(x, t) \in \partial \Omega \times[0, T] ; \quad u_{i}(x, t) \geq 0,(x, t) \in \Omega \times[-\tau, 0],
\end{gathered}
$$

where $b_{i j}(x, t), c_{i j}(x, t) \in C(\bar{\Omega} \times[0, T]), b_{i j} \geq 0$ for $(i \neq j)$, and $c_{i j} \geq 0$ for $i, j=1,2$, and $\tau_{2}=0$. Then

$$
u_{i}(x, t) \geq 0, \quad(x, t) \in \bar{\Omega} \times[0, T] .
$$

From Lemma 2.2 we easily get the following lemma.

Lemma 2.3. For any given $T>0$, if $u(x, t)$ and $v(x, t)$ belong to $C(\bar{\Omega} \times[0, T]) \cap C^{2,1}(\Omega \times$ $[0, T])$ and satisfy the relations

$$
\begin{aligned}
& \frac{\partial u}{\partial t}- D \Delta u-\left(a u(x, t-\tau)-\beta u^{2}(x, t)\right) \\
& \geq \frac{\partial v}{\partial t}-D \Delta v-\left(a v(x, t-\tau)-\beta v^{2}(x, t)\right), \quad x \in \Omega, t \in[0, T], \\
& \frac{\partial u}{\partial n} \geq \frac{\partial v}{\partial n}, \quad x \in \partial \Omega, t \in[0, T] ; \quad u(x, t)=\varphi(x, t) \geq v(x, t), \quad x \in \bar{\Omega}, t \in[-\tau, 0] .
\end{aligned}
$$

Then $u(x, t) \geq v(x, t)$.

Proof. Let $\omega(x, t)=u(x, t)-v(x, t)$, then

$$
\begin{aligned}
\frac{\partial \omega}{\partial t}-D \Delta \omega & \geq\left(a u(x, t-\tau)-\beta u^{2}(x, t)\right)-\left(a v(x, t-\tau)-\beta v^{2}(x, t)\right) \\
& =a \omega(x, t-\tau)-\beta \omega(x, t)(u(x, t)+v(x, t)) .
\end{aligned}
$$


Let $c_{11}=a, b_{11}=-\beta(u(x, t)+v(x, t))$. Since $c_{11}=a=\alpha e^{-\gamma \tau}>0$, by Lemma 2.2 we have $\omega(x, t) \geq 0$, that is,

$$
u(x, t) \geq v(x, t)
$$

Theorem 2.4. Let $u_{1}(x, t)$ and $u_{2}(x, t)$ be the solutions of system $(2.1)$ in $C(\bar{\Omega} \times[0, T]) \cap$ $C^{2,1}(\Omega \times[0, T])$, and if $f>d$, then

$$
\begin{gathered}
0 \leq u_{1}(x, t) \leq \max \left\{\left\|\varphi_{1}\right\|_{\infty}, a\right\} \stackrel{\text { def }}{=} M_{1}, \\
0 \leq u_{2}(x, t) \leq \max \left\{\left\|\varphi_{2}\right\|_{\infty}, \frac{M_{1}(f-d)}{d}\right\} \stackrel{\text { def }}{=} M_{2} .
\end{gathered}
$$

Proof. Let $0 \leq \sigma \leq T$. In order to investigate system (2.1), we firstly consider the following system:

$$
\begin{gathered}
\frac{\partial \psi_{1}}{\partial t}-D_{1} \Delta \psi_{1}=a \psi_{1}(x, t-\tau)+\psi_{1}(x, t)\left(-\psi_{1}(x, t)\right), \quad x \in \Omega, t \in[0, T] \\
\frac{\partial \psi_{2}}{\partial t}-D_{2} \Delta \psi_{2}=\psi_{2}(x, t)\left(-d+\frac{f \psi_{1}(x, t)}{\psi_{1}(x, t)+\psi_{2}(x, t)}\right), \quad x \in \Omega, t \in[0, T] \\
\frac{\partial \psi_{1}}{\partial n} \geq 0, \quad \frac{\partial \psi_{2}}{\partial n} \geq 0, \quad x \in \partial \Omega, t \in[0, T] \\
\psi_{1}(x, t) \geq 0, \quad \psi_{2}(x, 0) \geq 0, \quad x \in \bar{\Omega}, t \in[-\tau, 0]
\end{gathered}
$$

Since $a=\alpha e^{-\gamma \tau} \neq 0$ and $b_{12} \equiv 0$, by Lemma 2.2 we have

$$
u_{i}(x, t) \geq 0, \quad(x, t) \in \bar{\Omega} \times[0, \sigma] .
$$

Note that $\psi_{1}(x, t)$ is bounded in $\bar{\Omega} \times[0, \sigma]$ for any $\sigma(0<\sigma \leq T)$. If $\max _{\Omega \times[0, \sigma]} \psi_{1}(x, t) \geq$ $\left\|\varphi_{1}\right\|_{\infty}$, due to $\psi_{1}(x, t)$ satisfying the homogeneous Neumann boundary condition, there exists $\left(x_{0}, t_{0}\right) \in \Omega \times[0, \sigma]$ such that

$$
\psi_{1}\left(x_{0}, t_{0}\right)=\max _{\Omega \times[0, \sigma]} \psi_{1}(x, t) \geq\left\|\varphi_{1}\right\|_{\infty}
$$

Therefore, from the first equation of system (2.9) at the point $\left(x_{0}, t_{0}\right)$, we have

$$
\left.\left(a \psi_{1}(x, t-\tau)-\psi_{1}^{2}(x, t)\right)\right|_{\left(x_{0}, t_{0}\right)} \geq 0 .
$$

That is

$$
\psi_{1}\left(x_{0}, t_{0}\right) \leq a
$$

Hence, we obtain

$$
0 \leq \psi_{1}(x, t) \leq \max \left\{\left\|\varphi_{1}\right\|_{\infty}, a\right\}, \quad(x, t) \in \Omega \times[0, \sigma] .
$$


Taking the same argument in $[\sigma, 2 \sigma],[2 \sigma, 3 \sigma], \ldots,[(n-1) \sigma, n \sigma(=T)]$, we have

$$
0 \leq \psi_{1}(x, t) \leq M_{1}, \quad(x, t) \in \Omega \times[0, T] .
$$

Similarly, there exists $\left(x_{0}^{\prime}, t^{\prime}{ }_{0}\right) \in \Omega \times[0, T]$ such that

$$
\left.\left(\psi_{2}(x, t)\left(-d+\frac{f \psi_{1}(x, t)}{\psi_{1}(x, t)+\psi_{2}(x, t)}\right)\right)\right|_{\left(x^{\prime}, t^{\prime}{ }_{0}\right)} \geq 0 .
$$

Hence, if $f>d$, then

$$
0 \leq \psi_{2}(x, t) \leq \frac{M_{1}(f-d)}{d}
$$

By Lemma 2.3, we have

$$
u_{i}(x, t) \leq \psi_{i}(x, t), \quad i=1,2
$$

So we have

$$
\begin{gathered}
0 \leq u_{1}(x, t) \leq \max \left\{\left\|\varphi_{1}\right\|_{\infty}, a\right\}, \\
0 \leq u_{2}(x, t) \leq \max \left\{\left\|\varphi_{2}\right\|_{\infty}, \frac{M_{1}(f-d)}{d}\right\} .
\end{gathered}
$$

\section{Local asymptotic stability of the equilibria}

In this section, we discuss local asymptotic stability of the nonnegative equilibria by linearization method and analyzing the so-called characteristic equation of the equilibrium. It is obvious that system (2.1) only has three nonnegative equilibria: the equilibrium $E_{1}(0,0)$, the equilibrium $E_{2}(a, 0)$, and the positive equilibrium $E_{3}\left(c_{1}^{*}, c_{2}^{*}\right)$ when $f>d$ and $a / b>1-d / f$, where

$$
c_{1}^{*}=\frac{(a-b) f+b d}{f}, \quad c_{2}^{*}=\frac{(f-d) c_{1}^{*}}{d} .
$$

We will point out that $E_{1}(0,0)$ cannot be linearized though it is defined for system (2.1), so the local stability of $E_{1}(0,0)$ will be studied in another paper.

Let $\mu_{1}<\mu_{2}<\mu_{3}<\cdots<\mu_{n}<\cdots$ be the eigenvalues of the operator $-\Delta$ on $\Omega$ with the homogeneous Neumann boundary condition, and let $E\left(\mu_{i}\right)$ be the eigenfunction space corresponding to $\mu_{i}$ in $C^{1}(\Omega)$. It is well known that $\mu_{1}=0$ and the corresponding eigenfunction $\phi_{1}(x)>0$. Let $\left\{\phi_{i j} \mid j=1,2, \ldots, \operatorname{dimE}\left(\mu_{i}\right)\right\}$ be an orthogonal basis of $E\left(\mu_{i}\right), X=\left\{\mathbf{u}=\left(u_{1}, u_{2}\right) \mid \mathbf{u} \in\left[C^{1}(\Omega)\right]^{2}\right\}$ and $X_{i j}=\left\{\mathbf{c} \phi_{i j} \mid \mathbf{c} \in \mathbb{R}^{2}\right\}$, thus $X=\bigoplus_{i=1}^{\infty} X_{i}, X_{i}=$ $\bigoplus_{j=1}^{\operatorname{dimE}\left(\mu_{i}\right)} X_{i j}$ 
Let $u_{1}(x, t)=u_{1}^{*}(x, t)+c_{1}^{*}, u_{2}(x, t)=u_{2}^{*}(x, t)+c_{2}^{*}$, where $c_{1}^{*}$ and $c_{2}^{*}$ are both not zero. We still make $u_{1}(x, t), u_{2}(x, t)$ corresponding to $u_{1}^{*}(x, t), u_{2}^{*}(x, t)$, so the linearized equation of the system $(2.1)$ at $\left(c_{1}^{*}, c_{2}^{*}\right)$ is

$$
\begin{gathered}
\frac{\partial u_{1}}{\partial t}-D_{1} \Delta u_{1}=a u_{1}(x, t-\tau)-2 c_{1}^{*} u_{1}(x, t)-\frac{b\left(c_{2}^{*}\right)^{2}}{\left(c_{1}^{*}+c_{2}^{*}\right)^{2}} u_{1}(x, t)-\frac{b\left(c_{1}^{*}\right)^{2}}{\left(c_{1}^{*}+c_{2}^{*}\right)^{2}} u_{2}(x, t), \\
\frac{\partial u_{2}}{\partial t}-D_{2} \Delta u_{2}=\frac{f\left(c_{2}^{*}\right)^{2}}{\left(c_{1}^{*}+c_{2}^{*}\right)^{2}} u_{1}(x, t)-d u_{2}(x, t)+\frac{f\left(c_{1}^{*}\right)^{2}}{\left(c_{1}^{*}+c_{2}^{*}\right)^{2}} u_{2}(x, t), \quad x \in \Omega, t>0, \\
\frac{\partial u_{1}}{\partial n}=\frac{\partial u_{2}}{\partial n}=0, \quad x \in \partial \Omega, t>0, \\
u_{1}(x, t)=\varphi_{1}(x, t)-c_{1}^{*}, \quad u_{2}(x, t)=\varphi_{2}(x, 0)-c_{2}^{*}, \quad x \in \Omega, t \in[-\tau, 0] .
\end{gathered}
$$

From [5], we know that the characteristic equation for the system (3.2) is equivalent to

$$
\left|\begin{array}{cc}
\lambda+\mu_{k} D_{1}-a e^{-\lambda \tau}+2 c_{1}^{*}+\frac{b\left(c_{2}^{*}\right)^{2}}{\left(c_{1}^{*}+c_{2}^{*}\right)^{2}} & \frac{b\left(c_{1}^{*}\right)^{2}}{\left(c_{1}^{*}+c_{2}^{*}\right)^{2}} \\
-\frac{f\left(c_{2}^{*}\right)^{2}}{\left(c_{1}^{*}+c_{2}^{*}\right)^{2}} & \lambda+\mu_{k} D_{2}+d-\frac{f\left(c_{1}^{*}\right)^{2}}{\left(c_{1}^{*}+c_{2}^{*}\right)^{2}}
\end{array}\right|=0 .
$$

That is

$$
\begin{aligned}
& \left(\lambda+\mu_{k} D_{1}-a e^{-\lambda \tau}+2 c_{1}^{*}+\frac{b\left(c_{2}^{*}\right)^{2}}{\left(c_{1}^{*}+c_{2}^{*}\right)^{2}}\right)\left(\lambda+\mu_{k} D_{2}+d-\frac{f\left(c_{1}^{*}\right)^{2}}{\left(c_{1}^{*}+c_{2}^{*}\right)^{2}}\right) \\
& +\left(\frac{b\left(c_{1}^{*}\right)^{2}}{\left(c_{1}^{*}+c_{2}^{*}\right)^{2}}\right)\left(\frac{f\left(c_{2}^{*}\right)^{2}}{\left(c_{1}^{*}+c_{2}^{*}\right)^{2}}\right)=0 .
\end{aligned}
$$

3.1. Local asymptotic stability of the equilibrium $E_{2}(a, 0)$. From (3.4), it follows that at the equilibrium $E_{2}(a, 0)$,

$$
\left(\lambda+\mu_{k} D_{1}-a e^{-\lambda \tau}+2 a\right)\left(\lambda+\mu_{k} D_{2}+d-f\right)=0 .
$$

From the first factor of (3.5), we see

$$
\lambda+\mu_{k} D_{1}+2 a=a e^{-\lambda \tau} .
$$

Therefore,

$$
\left|\lambda+\mu_{k} D_{1}+2 a\right|=\left|a e^{-\lambda \tau}\right| .
$$

Now we will determine that all roots of (3.7) satisfy $\operatorname{Re} \lambda<0$. Suppose that there exists $\lambda_{0}$ such that $\operatorname{Re} \lambda_{0} \geq 0$. From (3.7), we deduce that

$$
\left|\lambda_{0}+\mu_{k} D_{1}+2 a\right| \leq|a|\left|e^{-\tau \operatorname{Re} \lambda_{0}}\right| \leq|a| .
$$


This implies that $\lambda_{0}$ is in the circle in the complex plane centered at $\left(-\left(\mu_{k} D_{1}+2 a\right), 0\right)$ and of radius $a$. However, as for given $\mu_{k}$ and $D_{1}$, it follows for ever that $\mu_{k} D_{1}+2 a>a$, therefore,

$$
\operatorname{Re} \lambda<0 .
$$

By the second factor of (3.5), we have

$$
\lambda=-\mu_{k} D_{2}-d+f \leq f-d
$$

If $f>d$, by taking $k=1\left(\mu_{1}=0\right)$, from (3.10), we obtain that there at least exists a root $\lambda_{0}$ of (3.5) such that $\operatorname{Re} \lambda_{0}>0$. Therefore, $E_{2}(a, 0)$ is unstable if the condition $f>d$ holds.

If $f<d$, then $f-d<0$, by (3.10), we have $\operatorname{Re} \lambda<0$. Therefore, if $f<d$, then $E_{2}(a, 0)$ is locally asymptotically stable.

3.2. Local asymptotic stability of the equilibrium $E_{3}\left(c_{1}^{*}, c_{2}^{*}\right)$. Let $\lambda=x+i y$, using (3.4), a direct calculation yields

$$
\begin{aligned}
& \left(x+i y+\mu_{k} D_{1}-a e^{-x \tau}(\cos (-y \tau)+i \sin (-y \tau))\right. \\
& \left.+2 c_{1}^{*}+\frac{b\left(c_{2}^{*}\right)^{2}}{\left(c_{1}^{*}+c_{2}^{*}\right)^{2}}\right)\left(x+i y+\mu_{k} D_{2}+d-\frac{f\left(c_{1}^{*}\right)^{2}}{\left(c_{1}^{*}+c_{2}^{*}\right)^{2}}\right) \\
& +\left(\frac{b\left(c_{1}^{*}\right)^{2}}{\left(c_{1}^{*}+c_{2}^{*}\right)^{2}}\right)\left(\frac{f\left(c_{2}^{*}\right)^{2}}{\left(c_{1}^{*}+c_{2}^{*}\right)^{2}}\right)=0,
\end{aligned}
$$

where $c_{1}^{*}=((a-b) f+b d) / f, c_{2}^{*}=\left((f-d) c_{1}^{*}\right) / d$.

Throughout the section we assume $f \geq 2 d$ and $a f \geq 2 b(f-d)$ and let

$$
\begin{gathered}
M_{1}=x+\mu_{k} D_{1}-a e^{-x \tau} \cos (-y \tau)+2 c_{1}^{*}+\frac{b\left(c_{2}^{*}\right)^{2}}{\left(c_{1}^{*}+c_{2}^{*}\right)^{2}}, \\
M_{2}=y+a e^{-x \tau} \sin (y \tau), \\
N_{1}=x+\mu_{k} D_{2}+d-\frac{f\left(c_{1}^{*}\right)^{2}}{\left(c_{1}^{*}+c_{2}^{*}\right)^{2}}, \\
N_{2}=y .
\end{gathered}
$$

Separating real and imaginary parts and applying (3.12) to (3.11), we obtain the equations

$$
\begin{gathered}
M_{1} N_{1}-M_{2} N_{2}+\left(\frac{b\left(c_{1}^{*}\right)^{2}}{\left(c_{1}^{*}+c_{2}^{*}\right)^{2}}\right)\left(\frac{f\left(c_{2}^{*}\right)^{2}}{\left(c_{1}^{*}+c_{2}^{*}\right)^{2}}\right)=0, \\
M_{1} N_{2}+M_{2} N_{1}=0 .
\end{gathered}
$$


Assume, for contradiction, that there exists a root $\lambda$ such that $\operatorname{Re} \lambda=x \geq 0$. By (3.12), we have

$$
\begin{aligned}
M_{1} & =x+\mu_{k} D_{1}-a e^{-x \tau} \cos (-y \tau)+2 c^{*} 1+\frac{b\left(c_{2}^{*}\right)^{2}}{\left(c_{1}^{*}+c_{2}^{*}\right)^{2}} \\
& \geq x+0-a e^{-x \tau} \cos (-y \tau)+2 c_{1}^{*}+\frac{b\left(c_{2}^{*}\right)^{2}}{\left(c_{1}^{*}+c_{2}^{*}\right)^{2}} \\
& \geq x-a+2 c_{1}^{*}+\frac{b\left(c_{2}^{*}\right)^{2}}{\left(c_{1}^{*}+c_{2}^{*}\right)^{2}} \\
& \geq\left(-a+c_{1}^{*}+\frac{b c_{2}^{*}}{c_{1}^{*}+c_{2}^{*}}\right)+\frac{b\left(c_{2}^{*}\right)^{2}}{\left(c_{1}^{*}+c_{2}^{*}\right)^{2}}+c_{1}^{*}-\frac{b c_{2}^{*}}{c_{1}^{*}+c_{2}^{*}} \\
& \geq \frac{b\left(c_{2}^{*}\right)^{2}}{\left(c_{1}^{*}+c_{2}^{*}\right)^{2}}+b(f-d) f-\frac{b c_{2}^{*}}{c_{1}^{*}+c_{2}^{*}}=\frac{b\left(c_{2}^{*}\right)^{2}}{\left(c_{1}^{*}+c_{2}^{*}\right)^{2}}>0, \\
N_{1} & =x+\mu_{k} D_{2}+d-\frac{f\left(c_{1}^{*}\right)^{2}}{\left(c_{1}^{*}+c_{2}^{*}\right)^{2}} \\
& =d-\frac{f c_{1}^{*}}{c_{1}^{*}+c_{2}^{*}}+\frac{f c_{1}^{*}}{c_{1}^{*}+c_{2}^{*}}-\frac{f\left(c_{1}^{*}\right)^{2}}{\left(c_{1}^{*}+c_{2}^{*}\right)^{2}} \geq \frac{f\left(c_{1}^{*}\right)^{2}}{\left(c_{1}^{*}+c_{2}^{*}\right)^{2}}>0 .
\end{aligned}
$$

Applying (3.15) and (3.16), one can obtain

$$
\left(\frac{b\left(c_{2}^{*}\right)^{2}}{\left(c_{1}^{*}+c_{2}^{*}\right)^{2}}\right)\left(\frac{f\left(c_{1}^{*}\right)^{2}}{\left(c_{1}^{*}+c_{2}^{*}\right)^{2}}\right) \leq M_{1} N_{1}
$$

Using (3.13) and (3.14), we have

$$
\begin{aligned}
& \left(\left(\frac{b\left(c_{1}^{*}\right)^{2}}{\left(c_{1}^{*}+c_{2}^{*}\right)^{2}}\right)\left(\frac{f\left(c_{2}^{*}\right)^{2}}{\left(c_{1}^{*}+c_{2}^{*}\right)^{2}}\right)\right)^{2} \\
& \quad=\left(M_{2} N_{2}\right)^{2}+\left(M_{1} N_{1}\right)^{2}+\left(M_{1} N_{2}\right)^{2}+\left(M_{2} N_{1}\right)^{2}
\end{aligned}
$$

If $N_{2} \neq 0$, by (3.15) and (3.18), we get

$$
\begin{aligned}
& \left(\left(\frac{b\left(c_{1}^{*}\right)^{2}}{\left(c_{1}^{*}+c_{2}^{*}\right)^{2}}\right)\left(\frac{f\left(c_{2}^{*}\right)^{2}}{\left(c_{1}^{*}+c_{2}^{*}\right)^{2}}\right)\right)^{2} \\
& \quad=\left(M_{2} N_{2}\right)^{2}+\left(M_{1} N_{1}\right)^{2}+\left(M_{1} N_{2}\right)^{2}+\left(M_{2} N_{1}\right)^{2}>\left(M_{1} N_{1}\right)^{2},
\end{aligned}
$$


it is a contradiction to (3.17). If $N_{2}=0$, from (3.12), we deduce $M_{2}=0$, again using (3.13), we have

$$
\begin{gathered}
\left(x+\mu_{k} D_{1}-a e^{-x \tau}+2 c^{*}{ }_{1}+\frac{b\left(c^{*}{ }_{2}\right)^{2}}{\left(c_{1}^{*}+c_{2}^{*}\right)^{2}}\right)\left(x+\mu_{k} D_{2}+d-\frac{f\left(c_{1}^{*}\right)^{2}}{\left(c_{1}^{*}+c_{2}^{*}\right)^{2}}\right) \\
+\left(\frac{b\left(c_{1}^{*}\right)^{2}}{\left(c_{1}^{*}+c_{2}^{*}\right)^{2}}\right)\left(\frac{f\left(c_{2}^{*}\right)^{2}}{\left(c_{1}^{*}+c_{2}^{*}\right)^{2}}\right)=0,
\end{gathered}
$$

that is,

$$
\begin{gathered}
\left(x+\mu_{k} D_{2}+\frac{f c_{1}^{*} c_{2}^{*}}{\left(c_{1}^{*}+c_{2}^{*}\right)^{2}}\right)\left(x+\mu_{k} D_{1}+a-a e^{-x \tau}+c_{1}^{*}-\frac{b c_{1}^{*} c_{2}^{*}}{\left(c_{1}^{*}+c_{2}^{*}\right)^{2}}\right) \\
+\left(\frac{b\left(c_{1}^{*}\right)^{2}}{\left(c_{1}^{*}+c_{2}^{*}\right)^{2}}\right)\left(\frac{f\left(c_{2}^{*}\right)^{2}}{\left(c_{1}^{*}+c_{2}^{*}\right)^{2}}\right)=0 .
\end{gathered}
$$

It is obvious that $x=-\mu_{k} D_{2}-f c_{1}^{*} c_{2}^{*} /\left(c_{1}^{*}+c_{2}^{*}\right)^{2}$ does not satisfy (3.21), so we have

$$
\begin{gathered}
\left(x+\mu_{k} D_{2}+\frac{f c_{1}^{*} c_{2}^{*}}{\left(c_{1}^{*}+c_{2}^{*}\right)^{2}}\right) \\
\times\left(x+\mu_{k} D_{1}+a-a e^{-x \tau}+c_{1}^{*}-\frac{b c_{1}^{*} c_{2}^{*}}{\left(c_{1}^{*}+c_{2}^{*}\right)^{2}}\right. \\
\left.+\frac{\left(b\left(c_{1}^{*}\right)^{2} /\left(c_{1}^{*}+c_{2}^{*}\right)^{2}\right)\left(f\left(c_{2}^{*}\right)^{2} /\left(c_{1}^{*}+c_{2}^{*}\right)^{2}\right)}{x+\mu_{k} D_{2}+f c_{1}^{*} c_{2}^{*} /\left(c_{1}^{*}+c_{2}^{*}\right)^{2}}\right)=0 .
\end{gathered}
$$

So all roots of (3.22) are given by (3.23), that is,

$$
\begin{aligned}
x & =-\mu_{k} D_{1}-a-c_{1}^{*}+a e^{-x \tau}+\frac{b c_{1}^{*} c_{2}^{*}}{\left(c_{1}^{*}+c_{2}^{*}\right)^{2}}-\frac{\left(b\left(c_{1}^{*}\right)^{2} /\left(c_{1}^{*}+c_{2}^{*}\right)^{2}\right)\left(f\left(c_{2}^{*}\right)^{2} /\left(c_{1}^{*}+c_{2}^{*}\right)^{2}\right)}{x+\mu_{k} D_{2}+f c_{1}^{*} c_{2}^{*} /\left(c_{1}^{*}+c_{2}^{*}\right)^{2}} \\
& \leq-c_{1}^{*}+\frac{b c_{1}^{*} c_{2}^{*}}{\left(c_{1}^{*}+c_{2}^{*}\right)^{2}}-\frac{\left(b\left(c_{1}^{*}\right)^{2} /\left(c_{1}^{*}+c_{2}^{*}\right)^{2}\right)\left(f\left(c_{2}^{*}\right)^{2} /\left(c_{1}^{*}+c_{2}^{*}\right)^{2}\right)}{x+\mu_{k} D_{2}+f c_{1}^{*} c_{2}^{*} /\left(c_{1}^{*}+c_{2}^{*}\right)^{2}} \\
& \leq-c_{1}^{*}+\frac{b c_{1}^{*} c_{2}^{*}}{\left(c_{1}^{*}+c_{2}^{*}\right)^{2}}\left(\frac{x+\mu_{k} D_{2}}{x+\mu_{k} D_{2}+f c_{1}^{*} c_{2}^{*} /\left(c_{1}^{*}+c_{2}^{*}\right)^{2}}\right) \\
& <-c_{1}^{*}+\frac{b c_{1}^{*} c_{2}^{*}}{\left(c_{1}^{*}+c_{2}^{*}\right)^{2}}<c_{1}^{*}\left(-1+\frac{b}{c_{1}^{*}+c_{2}^{*}}\right) \leq c_{1}^{*}\left(-1+\frac{b}{2 c_{1}^{*}}\right) \leq 0,
\end{aligned}
$$

it is a contradiction to $\operatorname{Re} \lambda=x \geq 0$. So we have that $\operatorname{Re} \lambda<0$ if $f \geq 2 d$ and $a f \geq 2 b(f-d)$, that is, the positive equilibrium $E_{3}\left(c_{1}^{*}, c_{2}^{*}\right)$ is locally asymptotically stable. 
From the above discussion, we can conclude the following.

Theorem 3.1. If $f \geq 2 d$ and $a f \geq 2 b(f-d)$, then the positive equilibrium $E_{3}\left(c_{1}^{*}, c_{2}^{*}\right)$ is locally asymptotically stable.

Theorem 3.2. If $f>d$, then the equilibrium $E_{2}(a, 0)$ is unstable.

Theorem 3.3. If $f<d$, then the equilibrium $E_{2}(a, 0)$ is locally asymptotically stable.

\section{Global asymptotic stability of the equilibria}

Note that $F_{1}\left(u_{1}(x, t), u_{2}(x, t), u_{1}(x, t-\tau)\right)$ and $F_{2}\left(u_{1}(x, t), u_{2}(x, t)\right)$, with respect to $u_{1}, u_{2}$, are continuous and mixed quasimonotone in $\Sigma \times \Sigma^{*}$, where $\Sigma, \Sigma^{*}$ are fixed and bounded subsets of $\mathbb{R}^{2}$. Thus there exist $K_{i} \geq 0(i=1,2)$ such that

$$
\begin{aligned}
& \left|F_{i}\left(u_{1}, u_{2}, u_{1}(x, t-\tau)\right)-F_{i}\left(u_{1}^{\prime}, u_{2}^{\prime}, u_{1}^{\prime}(x, t-\tau)\right)\right| \\
& \quad \leq K_{i}\left(\left|u_{1}(x, t)-u_{1}^{\prime}(x, t)\right|+u_{2}(x, t)-u_{2}^{\prime}(x, t) \mid\right),
\end{aligned}
$$

when $i=2, \tau=0$, where $\left(u_{1}, u_{1}^{\prime}\right),\left(u_{2}, u_{2}^{\prime}\right) \in \Sigma \times \Sigma^{*}$.

In order to investigate the dynamics of the system (2.1) we define two sequences of constant vectors $\left\{\overline{\mathbf{c}}^{(m)}\right\}=\left\{{\overline{c_{1}}}^{(m)},{\overline{c_{2}}}^{(m)}\right\}_{m=1}^{\infty},\left\{\underline{\mathbf{c}}^{(m)}\right\}=\left\{\underline{c}_{1}^{(m)}, \underline{\boldsymbol{c}}_{2}{ }^{(m)}\right\}_{m=1}^{\infty}$ satisfying the following relation:

$$
\begin{aligned}
& {\overline{c_{1}}}^{(m)}={\overline{c_{1}}}^{(m-1)}+\frac{1}{K_{1}}{\overline{c_{1}}}^{(m-1)}\left(a-{\overline{c_{1}}}^{(m-1)}-\frac{b \underline{\underline{c}}_{2}{ }^{(m-1)}}{{\overline{\bar{c}_{1}}}^{(m)}+\underline{\underline{c}}_{2}{ }^{(m-1)}}\right), \\
& \underline{c}_{1}^{(m)}=\underline{c}_{1}^{(m-1)}+\frac{1}{K_{1}} \underline{c}_{1}^{(m-1)}\left(a-\underline{c}_{1}^{(m-1)}-\frac{b{\overline{c_{2}}}^{(m-1)}}{\underline{c}_{1}^{(m-1)}+{\overline{c_{2}}}^{(m-1)}}\right), \\
& {\overline{c_{2}}}^{(m)}={\overline{c_{2}}}^{(m-1)}+\frac{1}{K_{2}}{\overline{c_{2}}}^{(m-1)}\left(-d+\frac{f{\overline{c_{1}}}^{(m)}}{{\overline{c_{1}}}^{(m)}+{\overline{c_{2}}}^{(m)}}\right), \\
& \underline{\underline{c}}_{2}{ }^{(m)}=\underline{\boldsymbol{c}}_{2}{ }^{(m-1)}+\frac{1}{K_{2}} \underline{\boldsymbol{c}}_{2}{ }^{(m-1)}\left(-d+\frac{f \underline{\underline{c}}_{2}{ }^{(m-1)}}{\underline{\boldsymbol{c}}_{2}{ }^{(m-1)}+\underline{\boldsymbol{c}}_{2}{ }^{(m-1)}}\right), \\
& \overline{\mathbf{c}}^{(0)}=\tilde{\mathbf{c}}, \quad \underline{\mathbf{c}}^{(0)}=\widehat{\mathbf{c}}, \quad m=1,2, \ldots,
\end{aligned}
$$

where $(\tilde{\mathbf{c}}, \widehat{\mathbf{c}})$ is a pair of coupled upper and lower solutions of system (2.1). It is easy to prove the following lemma.

Lemma 4.1. The sequences $\left\{\overline{\mathbf{c}}^{(m)}\right\},\left\{\underline{\mathbf{c}}^{(m)}\right\}$ given by (4.2) with $\overline{\mathbf{c}}^{(0)}=\tilde{\mathbf{c}}$ and $\underline{\mathbf{c}}^{(0)}=\hat{\mathbf{c}}$ possess the monotone property

$$
\widehat{\mathbf{c}} \leq \underline{\mathbf{c}}^{(m)} \leq \underline{\mathbf{c}}^{(m+1)} \leq \overline{\mathbf{c}}^{(m+1)} \leq \overline{\mathbf{c}}^{(m)} \leq \widetilde{\mathbf{c}}, \quad m=1,2, \ldots
$$


Proof. Since $(\tilde{\mathbf{c}}, \widehat{\mathbf{c}})$ is a pair of coupled upper and lower solutions of the system (2.1), so it follows from (2.2) that

$$
\begin{aligned}
& {\overline{c_{1}}}^{(0)}-{\overline{c_{1}}}^{(1)}={\overline{c_{1}}}^{(0)}-\left({\overline{c_{1}}}^{(0)}+\frac{1}{K_{1}}{\overline{c_{1}}}^{(0)}\left(a-{\overline{c_{1}}}^{(0)}-\frac{b \underline{c}_{2}{ }^{(0)}}{{\overline{c_{1}}}^{(0)}+\underline{c}_{2}{ }^{(0)}}\right)\right) \\
& =-\frac{1}{K_{1}}{\overline{c_{1}}}^{(0)}\left(a-{\overline{c_{1}}}^{(0)}-\frac{b \underline{c}_{2}{ }^{(0)}}{{\overline{c_{1}}}^{(0)}+\underline{c}_{2}{ }^{(0)}}\right) \geq 0, \\
& \underline{c}_{1}{ }^{(1)}-\underline{c}_{1}{ }^{(0)}=\left(\underline{c}_{1}{ }^{(0)}+\frac{1}{K_{1}} \underline{c}_{1}{ }^{(0)}\left(a-\underline{c}_{1}{ }^{(0)}-\frac{b \overline{\bar{c}_{2}}{ }^{(0)}}{\underline{c}_{1}{ }^{(0)}+\overline{c_{2}}{ }^{(0)}}\right)\right)-\underline{c}_{1}{ }^{(0)} \\
& =\frac{1}{K_{1}} \underline{c}_{1}{ }^{(0)}\left(a-\underline{c}_{1}{ }^{(0)}-\frac{b \overline{\bar{c}_{2}}}{{ }^{(0)}}\right) \geq 0 .
\end{aligned}
$$

This gives ${\overline{\bar{c}_{1}}}^{(0)} \geq{\overline{\bar{c}_{1}}}^{(1)}$ and $\underline{\underline{c}}_{1}{ }^{(1)} \geq \underline{\underline{c}}_{1}{ }^{(0)}$. Similarly by (2.2) and the quasimonotone property, we have

$$
\begin{aligned}
K_{1}\left({\overline{c_{1}}}^{(1)}-\underline{c}_{1}{ }^{(1)}\right)= & K_{1}\left({\overline{c_{1}}}^{(0)}-\underline{c}_{1}{ }^{(0)}\right)+{\overline{c_{1}}}^{(0)}\left(a-{\overline{c_{1}}}^{(0)}-\frac{b \underline{c}_{2}{ }^{(0)}}{\overline{\bar{c}_{1}}{ }^{(0)}+\underline{c}_{2}{ }^{(0)}}\right) \\
& -\underline{c}_{1}{ }^{(0)}\left(a-\underline{c}_{1}{ }^{(0)}-\frac{b \overline{c_{2}}}{{\underline{c_{1}}}^{(0)}+{\overline{c_{2}}}^{(0)}}\right) \geq 0 .
\end{aligned}
$$

This yields ${\overline{c_{1}}}^{(1)} \geq \underline{c}_{1}{ }^{(1)}$. The above conclusions show that

$$
\underline{\mathbf{c}}^{(0)} \leq \underline{\mathbf{c}}^{(1)} \leq \overline{\mathbf{c}}^{(1)} \leq \overline{\mathbf{c}}^{(0)}
$$

Assume, by induction, that $\underline{\mathbf{c}}^{(m-1)} \leq \underline{\mathbf{c}}^{(m)} \leq \overline{\mathbf{c}}^{(m)} \leq \overline{\mathbf{c}}^{(m-1)}$ for some $m>1$, Then by (4.6) and (2.2), we have

$$
\begin{aligned}
K_{1}\left({\overline{c_{1}}}^{(m)}-\underline{\underline{c}}_{1}{ }^{(m+1)}\right) \\
=K_{1}\left({\overline{c_{1}}}^{(m-1)}-\underline{\underline{c}}_{1}{ }^{(m)}\right)+{\overline{c_{1}}}^{(m-1)}\left(a-{\overline{c_{1}}}^{(m-1)}-\frac{b \underline{c}_{2}{ }^{(m-1)}}{{\overline{c_{1}}}^{(m-1)}+\underline{c}_{2}{ }^{(m-1)}}\right) \\
\quad-\underline{c}_{1}{ }^{(m)}\left(a-\underline{c}_{1}{ }^{(m)}-\frac{b{\overline{c_{2}}}^{(m)}}{{\underline{c_{1}}}^{(m)}+{\overline{c_{2}}}^{(m)}}\right) \geq 0 .
\end{aligned}
$$

This yields ${\overline{\bar{c}_{1}}}^{(m)} \geq \underline{\boldsymbol{c}}_{1}{ }^{(m+1)}$. A similar argument gives $\underline{\mathbf{c}}^{(m)} \leq \underline{\mathbf{c}}^{(m+1)} \leq \overline{\mathbf{c}}^{(m+1)} \leq \overline{\mathbf{c}}^{(m)}$. A similar argument gives $\overline{\bar{c}_{2}}{ }^{(m)}$ and $\underline{\underline{c}}_{2}{ }^{(m)}$. Therefore, the monotone property (4.3) follows by the principle of induction.

By monotone bounds principle, we get

$$
\lim _{m \rightarrow \infty} \underline{\mathbf{c}}^{(m)}=\underline{\mathbf{c}}, \quad \lim _{m \rightarrow \infty} \overline{\mathbf{c}}^{(m)}=\overline{\mathbf{c}},
$$


with

$$
\widehat{\mathbf{c}} \leq \underline{\mathbf{c}}^{(m)} \leq \underline{\mathbf{c}}^{(m+1)} \leq \underline{\mathbf{c}} \leq \overline{\mathbf{c}} \leq \overline{\mathbf{c}}^{(m+1)} \leq \overline{\mathbf{c}}^{(m)} \leq \widetilde{\mathbf{c}} .
$$

In (4.2), letting $m \rightarrow \infty$, we have

$$
\begin{array}{cl}
\overline{c_{1}}\left(a-\overline{c_{1}}-\frac{b \underline{c}_{2}}{\overline{c_{1}}+\underline{c}_{2}}\right)=0, & \underline{c}_{1}\left(a-\underline{c}_{1}-\frac{b \overline{c_{2}}}{\underline{c}_{1}+\overline{c_{2}}}\right)=0, \\
\overline{c_{2}}\left(-d+\frac{f \overline{c_{1}}}{\overline{c_{1}}+\overline{c_{2}}}\right)=0, & \underline{c}_{2}\left(-d+\frac{f \underline{c}_{1}}{\underline{c}_{1}+\underline{c}_{2}}\right)=0 .
\end{array}
$$

Considering [16, Theorem 2.2] as a corollary, we obtain the following.

Theorem 4.2. Let $\mathbf{c}, \overline{\mathbf{c}}$ be the limits in (4.2), then for any initial function $\varphi=\left(\varphi_{1}, \varphi_{2}\right)$ in $\langle\underline{\mathbf{c}}, \overline{\mathbf{c}}\rangle$ the solution of system (2.1) satisfies the relation

$$
\underline{\mathbf{c}} \leq \mathbf{u}(x, t) \leq \overline{\mathbf{c}} \text { as } t \rightarrow \infty .
$$

Moreover, if, in addition, $\underline{\mathbf{c}}=\overline{\mathbf{c}}\left(\equiv \mathbf{c}^{*}\right)$, then $\mathbf{c}^{*}$ is the unique global solution of the system (2.1) in $\langle\underline{\mathbf{c}}, \overline{\mathbf{c}}\rangle$, and

$$
\lim _{t \rightarrow \infty} \mathbf{u}(x, t)=\mathbf{c}^{*}, \quad x \in \Omega
$$

Theorem 4.3. Let $\left(u_{1}(x, t), u_{2}(x, t)\right)$ be the solution of the system $(2.1)$, if $\varphi_{1}(x, 0) \neq 0$ and $\varphi_{2}(x, 0) \equiv 0$, then $u_{1}(x, t)>0, u_{2}(x, t) \equiv 0$, and

$$
\left(u_{1}(x, t), u_{2}(x, t)\right) \longrightarrow(a, 0), \quad t \longrightarrow+\infty .
$$

Proof. By the standard maximum principle for parabolic boundary-value problems with homogeneous Neumann boundary condition, we see that

$$
\left(u_{1}(x, t), u_{2}(x, t)\right) \geq(0,0) \text {. }
$$

It is obvious that if $\varphi_{1}(x, 0) \neq 0, \varphi_{2}(x, 0) \equiv 0$, then

$$
u_{1}(x, t)>0, \quad u_{2}(x, t) \equiv 0 .
$$

Now, the system (2.1) becomes the scalar boundary-value problem

$$
\begin{gathered}
\frac{\partial u_{1}}{\partial t}-D_{1} \Delta u_{1}=a u_{1}(x, t-\tau)-u_{1}^{2}(x, t), \quad x \in \Omega, t>0 \\
\frac{\partial u_{1}}{\partial n}=0, \quad x \in \partial \Omega, t>0 ; \quad u_{1}(x, t)=\varphi_{1}(x, t) \geq 0, \quad x \in \Omega, t \in[-\tau, 0] .
\end{gathered}
$$

By continuity and Lemma 2.3, it follows that there exist $\delta>0$ and $t^{*}>0$ satisfying

$$
u_{1}(x, t) \geq \delta, \quad(x, t) \in\left[t^{*}, t^{*}+\tau\right] .
$$


Let $\widetilde{\mathbf{c}}=M, \widehat{\mathbf{c}}=\varepsilon \geq 0$, where

$$
M=\max \left\{\max _{\Omega \times\left[t^{*}, t^{*}+\tau\right]} u_{1}(x, t), a\right\}, \quad \varepsilon=\min \{a, \delta\} .
$$

Then

$$
\begin{gathered}
\varepsilon \leq a \Longrightarrow \varepsilon^{2} \leq a \varepsilon \Longrightarrow a \varepsilon-\varepsilon^{2} \geq 0, \\
a \leq M \Longrightarrow a M \leq M^{2} \Longrightarrow a M-M^{2} \leq 0 .
\end{gathered}
$$

Therefore, $(\tilde{\mathbf{c}}, \widehat{\mathbf{c}})$ is a pair of strong upper and lower solutions of the system (4.17). Applying Theorem 4.2 and (4.3), we obtain that there exist $\underline{\mathbf{c}}, \overline{\mathbf{c}}$ such that

$$
\begin{gathered}
\varepsilon \leq \hat{\mathbf{c}} \leq \underline{\mathbf{c}} \leq \overline{\mathbf{c}} \leq \tilde{\mathbf{c}} \leq M, \\
\underline{c}(a-\underline{c})=0, \quad \bar{c}(a-\bar{c})=0,
\end{gathered}
$$

while the equation $c(a-c)=0$ has a unique positive solution $c^{*}=a$.

Therefore

$$
\bar{c}=\underline{c}=c^{*}=a, \quad \lim _{t \rightarrow \infty} \mathbf{u}_{1}(x, t)=\mathbf{c}^{*}=a .
$$

THeORem 4.4. If $f \geq 2 d$ and $a f \geq 2 b(f-d)$, then for any nonnegative initial function $\varphi=\left(\varphi_{1}, \varphi_{2}\right)$ the system (2.1) has a unique global solution with

$$
\left(u_{1}(x, t), u_{2}(x, t)\right) \longrightarrow\left(c_{1}^{*}, c_{2}^{*}\right) \text { as } t \longrightarrow+\infty \text {. }
$$

Proof. Let $\omega_{1}(x, t)$ be the solution of the scalar boundary-value problem

$$
\begin{gathered}
\frac{\partial \omega_{1}}{\partial t}-D_{1} \Delta \omega_{1}=a \omega_{1}(x, t-\tau)-\omega_{1}^{2}(x, t), \quad x \in \Omega, t>0 \\
\frac{\partial \omega_{1}}{\partial n}=0, \quad x \in \partial \Omega, t>0 \\
\omega_{1}(x, t)=\varphi_{1}(x, t) \geq 0, \quad x \in \Omega, t \in[-\tau, 0] .
\end{gathered}
$$

Applying Lemma 2.3, we have

$$
u_{1}(x, t) \leq \omega_{1}(x, t), \quad(x, t) \in \Omega \times[0,+\infty] .
$$

By Theorem 4.3, it follows that

$$
\omega_{1}(x, t) \longrightarrow a, \quad t \longrightarrow \infty
$$

Hence, for any $\varepsilon>0$, there always exists $t^{* *}>0$ as $t>t^{* *}$ such that

$$
u_{1}(x, t) \leq \omega_{1}(x, t) \leq a+\varepsilon .
$$


Let $\omega_{2}(x, t)$ be the solution of the scalar boundary-value problem

$$
\begin{aligned}
& \frac{\partial \omega_{2}}{\partial t}-D_{2} \Delta \omega_{2}=-d \omega_{2}(x, t)+\frac{f(a+\varepsilon) \omega_{2}(x, t)}{(a+\varepsilon)+\omega_{2}(x, t)} \\
& \leq-d \omega_{2}(x, t)+f(a+\varepsilon), \quad(x, t) \in \Omega \times\left[t^{* *},+\infty\right], \\
& \frac{\partial \omega_{2}}{\partial n}=0, \quad(x, t) \in \partial \Omega \times\left[t^{* *},+\infty\right], \\
& \omega_{2}(x, t)=\varphi_{1}\left(x, t^{* *}\right) \geq 0, \quad x \in \Omega .
\end{aligned}
$$

By the quasimonotone property of $F_{2}\left(u_{1}, u_{2}\right)$, we obtain

$$
u_{2}(x, t) \leq \omega_{2}(x, t), \quad(x, t) \in \Omega \times\left[t^{* *},+\infty\right] .
$$

Now we consider the scalar boundary-value problem

$$
\begin{gathered}
\frac{\partial \omega_{2}}{\partial t}-D_{2} \Delta \omega_{2}=-d \omega_{2}(x, t)+f(a+\varepsilon), \quad(x, t) \in \Omega \times\left[t^{* *},+\infty\right], \\
\frac{\partial \omega_{2}}{\partial n}=0, \quad(x, t) \in \partial \Omega \times\left[t^{* *},+\infty\right], \\
\omega_{2}(x, t)=\varphi_{1}\left(x, t^{* *}\right) \geq 0, \quad x \in \Omega .
\end{gathered}
$$

By [22], we have $\omega_{2}(x, t) \rightarrow f(a+\varepsilon) / d$ as $t$ sufficiently large. Therefore, for the above given $\varepsilon$, there exists $t_{0}>0$ satisfying

$$
u_{2}(x, t) \leq \frac{f(a+\varepsilon)}{d}, \quad(x, t) \in \Omega \times\left[t_{0},+\infty\right] .
$$

Let

$$
\begin{gathered}
\tilde{c_{1}}=a+\varepsilon, \quad \hat{c_{1}}=\delta, \\
\tilde{c_{2}}=\frac{f(a+\varepsilon)}{d}, \quad \hat{c_{2}}=\delta,
\end{gathered}
$$

where $\varepsilon$ and $\delta$ are sufficiently small positive constants.

By $f \geq 2 d$ and $a f \geq 2 b(f-d)$, applying Lemmas 2.2 and 2.3 , for $t$ sufficiently large, we obtain that

$$
\begin{aligned}
& \hat{c_{1}} \leq u_{1}(x, t) \leq \tilde{c_{1}}, \quad \hat{c_{2}} \leq u_{2}(x, t) \leq \tilde{c_{2}}, \\
& \widetilde{c_{2}}=\frac{f(a+\varepsilon)}{d} \Longrightarrow d \tilde{c_{2}}=f(a+\varepsilon) \Longrightarrow d \widetilde{c_{2}} \geq \frac{f(a+\varepsilon) \tilde{c_{2}}}{\widetilde{c_{1}}+\widetilde{c_{2}}} \Longrightarrow-d \tilde{c_{2}}+\frac{f(a+\varepsilon) \widetilde{c_{2}}}{\widetilde{c_{1}}+\widetilde{c_{2}}} \leq 0 \\
& \widetilde{c_{1}}=a+\varepsilon \geq a \Longrightarrow{\tilde{c_{1}}}^{2} \geq a \tilde{c_{1}} \Longrightarrow 0 \geq a \tilde{c_{1}}-{\tilde{c_{1}}}^{2} \Longrightarrow a \tilde{c_{1}}-{\tilde{c_{1}}}^{2}-\frac{b \delta \tilde{c_{1}}}{\delta+\widetilde{c_{1}}} \leq 0 \text {. }
\end{aligned}
$$

Let $\varepsilon$ and $\delta$ be sufficiently small positive constants. Applying $f \geq 2 d$ and $a f \geq 2 b(f-d)$, it is not difficult to prove that $\widehat{c_{1}}=\delta_{1}$ and $\widehat{c_{2}}=\delta_{2}$ satisfy (2.2). 
Hence, $\left(\tilde{c_{1}}, \widetilde{c_{2}}\right),\left(\widehat{c_{1}}, \hat{c_{2}}\right)$ are a pair of coupled upper and lower solutions of the system (2.1).

By Theorem 4.2, we obtain that $\underline{\mathbf{c}}$ and $\overline{\mathbf{c}}$ satisfy

$$
\begin{gathered}
0<\hat{\mathbf{c}} \leq \underline{\mathbf{c}} \leq \overline{\mathbf{c}} \leq \tilde{\mathbf{c}}, \\
\overline{c_{1}}\left(a-\overline{c_{1}}-\frac{b \underline{c}_{2}}{\overline{c_{1}}+\underline{c}_{2}}\right)=0, \\
\underline{c}_{1}\left(a-\underline{c}_{1}-\frac{b \overline{c_{2}}}{\underline{c_{1}}+\overline{c_{2}}}\right)=0, \\
\overline{c_{2}}\left(-d+\frac{f \overline{c_{1}}}{\overline{c_{1}}+\overline{c_{2}}}\right)=0, \\
\underline{c}_{2}\left(-d+\frac{f \underline{c}_{1}}{{\underline{c_{1}}}_{1}+\underline{c}_{2}}\right)=0 .
\end{gathered}
$$

Since $f \geq 2 d$ and $a f \geq 2 b(f-d)$ and the following equations have unique positive solutions:

$$
c_{1}\left(a-c_{1}-\frac{b c_{2}}{c_{1}+c_{2}}\right)=0, \quad c_{2}\left(-d+\frac{f c_{1}}{c_{1}+c_{2}}\right)=0,
$$

therefore

$$
\overline{c_{1}}=\underline{c}_{1}=c_{1}^{*}, \quad \overline{c_{2}}=\underline{c}_{2}=c_{2}{ }^{*},
$$

where

$$
c^{*}{ }_{1}=\frac{(a-b) f+b d}{f}, \quad c^{*}{ }_{2}=\frac{(f-d) c^{*}{ }_{1}}{d} .
$$

Therefore

$$
\lim _{t \rightarrow+\infty} u_{1}(x, t)=c_{1}^{*}, \quad \lim _{t \rightarrow+\infty} u_{2}(x, t)=c_{2}^{*} .
$$

THeOREM 4.5. If $f<d$, then for any nonnegative initial function $\varphi_{i}(x, t)(i=1,2)$, the system (2.1) has a unique global nonnegative solution $\left(u_{1}(x, t), u_{2}(x, t)\right)$ satisfying

$$
\left(u_{1}(x, t), u_{2}(x, t)\right) \longrightarrow(a, 0), \quad t \longrightarrow+\infty .
$$

Proof. Let $c_{1}{ }^{*}$ and $c_{2}{ }^{*}$ be the solutions of the following system:

$$
\begin{gathered}
c_{1}^{*}\left(a-c_{1}^{*}-\frac{b c_{2}^{*}}{c_{1} *+c_{2} *}\right)=0, \\
c_{2}^{*}\left(-d+\frac{f c_{1}^{*}}{c_{1} *+c_{2} *}\right)=0 .
\end{gathered}
$$


18 A ratio-dependent predator-prey system

If $f<d$, then (4.40) has only one nonzero solution and one nonnegative solution:

$$
c_{1}^{*}=a, \quad c_{2}^{*}=0 .
$$

Let $\widetilde{c_{2}}$ be sufficiently large and let $0<\delta(\leq a)$ be sufficiently small, and $\tilde{c_{1}}, \hat{c_{1}}, \hat{c_{2}}$ satisfy

$$
\tilde{c_{1}} \geq a, \quad \hat{c_{1}}=\delta, \quad \hat{c_{2}}=0
$$

Applying Lemmas 2.2 and 2.3, if the condition $f<d$ holds, it is easy to prove

$$
\hat{c_{1}} \leq u_{1}(x, t) \leq \tilde{c_{1}}, \quad \hat{c_{2}} \leq u_{2}(x, t) \leq \tilde{c_{2}},
$$

and $\left(\tilde{c_{1}}, \tilde{c_{2}}\right),\left(\widehat{c_{1}}, \hat{c_{2}}\right)$ satisfying $(2.2)$. Hence, $\left(\tilde{c_{1}}, \tilde{c_{2}}\right),\left(\widehat{c_{1}}, \widehat{c_{2}}\right)$ are a pair of coupled upper and lower solutions of the system (2.1). By Theorem 4.2, we obtain $\underline{\mathbf{c}}$ and $\overline{\mathbf{c}}$ satisfying

$$
\begin{gathered}
0<\hat{\mathbf{c}} \leq \underline{\mathbf{c}} \leq \overline{\mathbf{c}} \leq \tilde{\mathbf{c}}, \\
\overline{c_{1}}\left(a-\overline{c_{1}}-\frac{b \underline{c}_{2}}{\overline{c_{1}}+\underline{c}_{2}}\right)=0, \\
\underline{c}_{1}\left(a-\underline{c}_{1}-\frac{b \overline{c_{2}}}{\underline{c_{1}}+\overline{c_{2}}}\right)=0, \\
\overline{c_{2}}\left(-d+\frac{f \overline{c_{1}}}{\overline{c_{1}}+\overline{c_{2}}}\right)=0, \\
\underline{c}_{2}\left(-d+\frac{f \underline{c}_{2}}{{\underline{c_{1}}}_{1}+\underline{c}_{2}}\right)=0 .
\end{gathered}
$$

We see from $\underline{c}_{2}{ }^{(0)}=0$ that $\underline{c}_{2}{ }^{(m)}=0$ for every $m=1,2, \ldots$. This implies $\underline{c}_{2}=0$.

Using $0<\delta \leq \underline{c}_{1} \leq \overline{c_{1}}$, we have $\underline{c}_{1}=a$. Since $f<d$, and

$$
\begin{gathered}
\overline{c_{1}}\left(a-\overline{c_{1}}-\frac{b \underline{c}_{2}}{\overline{c_{1}}+\underline{c}_{2}}\right)=0, \\
\underline{c}_{1}\left(a-\underline{c}_{1}-\frac{b \overline{c_{2}}}{\overline{c_{1}}+\overline{c_{2}}}\right)=0, \\
\overline{c_{2}}\left(-d+\frac{f \overline{c_{2}}}{\overline{c_{1}}+\overline{c_{2}}}\right)=0,
\end{gathered}
$$

so we have

$$
\overline{c_{1}}=\underline{c}_{1}=a, \quad \overline{c_{2}}=\underline{c}_{2}=0 .
$$

By Theorem 4.2, for any nonnegative initial function $\varphi_{i}(x, t), i=1,2$, the system $(2.1)$ has a unique global nonnegative solution $\left(u_{1}(x, t), u_{2}(x, t)\right)$ in $\varphi_{i}(x, t) \in\langle\hat{\mathbf{c}}, \tilde{\mathbf{c}}\rangle$ satisfying

$$
\left(u_{1}(x, t), u_{2}(x, t)\right) \longrightarrow(a, 0), \quad t \longrightarrow+\infty .
$$




\section{Acknowledgments}

This work is supported by the National Natural Science Foundation of China (No. 10471117), the Henan Innovation Project for the University Prominent Research Talents (No. 2005KYCX017), and the Scientific Research Foundation for the Returned Overseas Chinese Scholars, State Education Ministry. We would like to thank the referees and the editor for their careful reading of the original manuscript and their many valuable comments and suggestions that greatly improved the presentation of this work.

\section{References}

[1] W. G. Aiello and H. I. Freedman, A time-delay model of single-species growth with stage structure, Mathematical Biosciences 101 (1990), no. 2, 139-153.

[2] W. G. Aiello, H. I. Freedman, and J. Wu, Analysis of a model representing stage-structured population growth with state-dependent time delay, SIAM Journal on Applied Mathematics 52 (1992), no. 3, 855-869.

[3] J. F. M. Al-Omari and S. A. Gourley, Stability and traveling fronts in Lotka-Volterra competition models with stage structure, SIAM Journal on Applied Mathematics 63 (2003), no. 6, 2063-2086.

[4] H. Amann, Dynamic theory of quasilinear parabolic equations II: reaction-diffusion systems, Differential and Integral Equations 3 (1990), no. 1, 13-75.

[5] T. Faria, Stability and bifurcation for a delayed predator-prey model and the effect of diffusion, Journal of Mathematical Analysis and Applications 254 (2001), no. 2, 433-463.

[6] H. I. Freedman, Deterministic Mathematical Models in Population Ecology, Monographs and Textbooks in Pure and Applied Mathematics, vol. 57, Marcel Dekker, New York, 1980.

[7] H. I. Freedman and J. Wu, Persistence and global asymptotic stability of single species dispersal models with stage structure, Quarterly of Applied Mathematics 49 (1991), no. 2, 351-371.

[8] S. A. Gourley and Y. Kuang, A stage structured predator-prey model and its dependence on maturation delay and death rate, Journal of Mathematical Biology 49 (2004), no. 2, 188-200.

[9] Y. Kuang, Delay Differential Equations with Applications in Population Dynamics, Mathematics in Science and Engineering, vol. 191, Academic Press, Massachusetts, 1993.

[10] Y. Kuang and E. Beretta, Global qualitative analysis of a ratio-dependent predator-prey system, Journal of Mathematical Biology 36 (1998), no. 4, 389-406.

[11] J. D. Murray, Mathematical Biology, Biomathematics, vol. 19, Springer, Berlin, 1989.

[12] P. Y. H. Pang and M. Wang, Strategy and stationary pattern in a three-species predator-prey model, Journal of Differential Equations 200 (2004), no. 2, 245-273.

[13] C. V. Pao, Nonlinear Parabolic and Elliptic Equations, Plenum Press, New York, 1992.

[14] _ Dynamics of nonlinear parabolic systems with time delays, Journal of Mathematical Analysis and Applications 198 (1996), no. 3, 751-779.

[15] _ Systems of parabolic equations with continuous and discrete delays, Journal of Mathematical Analysis and Applications 205 (1997), no. 1, 157-185.

[16] _ Convergence of solutions of reaction-diffusion systems with time delays, Nonlinear Analysis. Series A: Theory and Methods 48 (2002), no. 3, 349-362.

[17] S. G. Ruan and J. Wu, Reaction-diffusion equations with infinite delay, The Canadian Applied Mathematics Quarterly 2 (1994), no. 4, 485-550.

[18] J. W.-H. So, J. Wu, and X. Zou, A reaction-diffusion model for a single species with age structure. I. Traveling fronts on unbounded domains, Proceedings of the Royal Society of London. Series A 457 (2001), no. 2012, 1841-1853.

[19] X. Song, L. Cai, and A. U. Neumann, Ratio-dependent predator-prey system with stage structure for prey, Discrete and Continuous Dynamical Systems. Series B 4 (2004), no. 3, 747-758. 


\section{A ratio-dependent predator-prey system}

[20] X. Song and L. Chen, Optimal harvesting and stability for a predator-prey system with stage structure, Acta Mathematical Application Sinica 18 (2002), no. 3, 307-314.

[21] Y. Takeuchi, Global Dynamical Properties of Lotka-Volterra Systems, World Scientific, New Jersey, 1996.

[22] M. Wang, Nonlinear Partial Differential Equations of Parabolic Type, Science Press, Beijing, 1993.

Xinyu Song: Department of Mathematics, Xinyang Normal University, Xinyang 464000, Henan, China

E-mail address: xysong88@163.com

Zhihao Ge: Faculty of Science, Xi' an Jiaotong University, Xi'an 710049, China

E-mail address: zhihaoge@gmail.com

Jingang Wu: Department of Mathematics, Xinyang Normal University, Xinyang 464000, Henan, China

E-mail address: kyczrk@mail2.xytc.edu.cn 


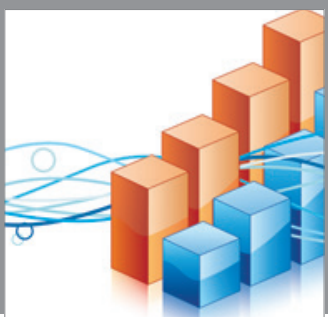

Advances in

Operations Research

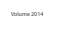

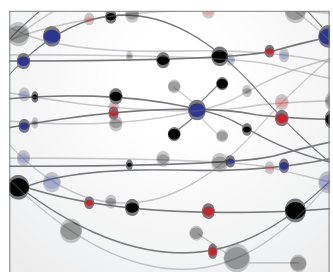

\section{The Scientific} World Journal
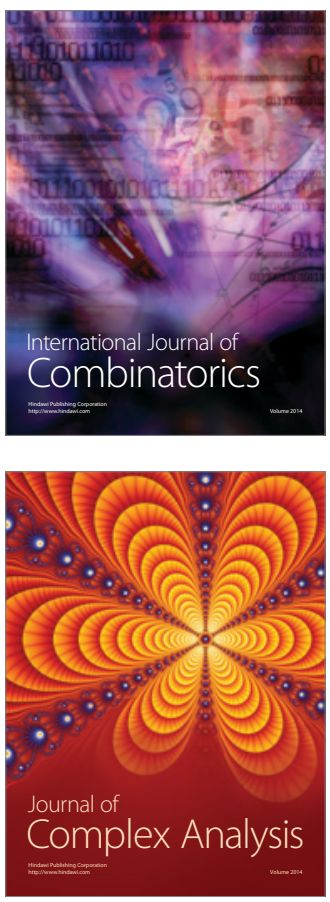

International Journal of

Mathematics and

Mathematical

Sciences
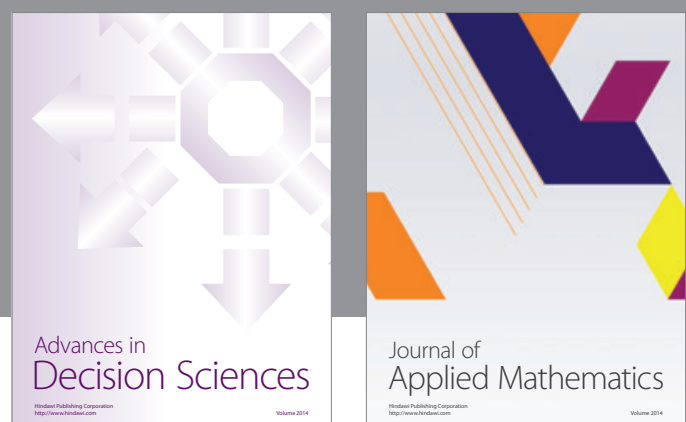

Journal of

Applied Mathematics
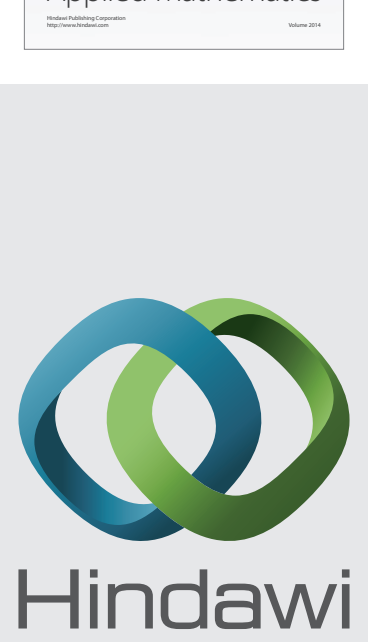

Submit your manuscripts at http://www.hindawi.com
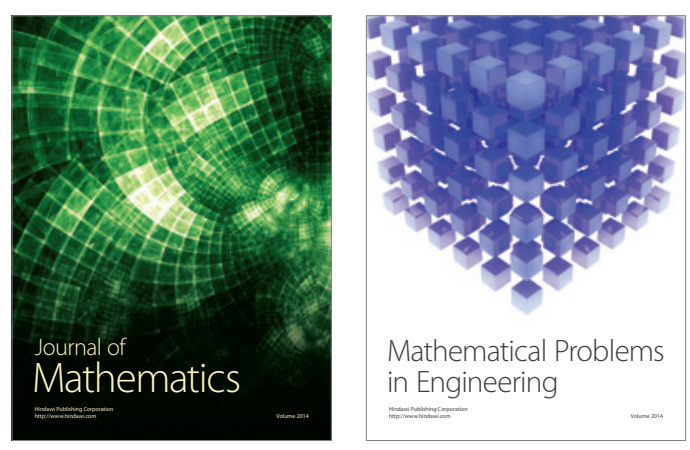

Mathematical Problems in Engineering
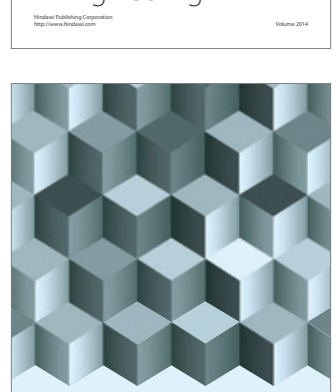

Journal of

Function Spaces
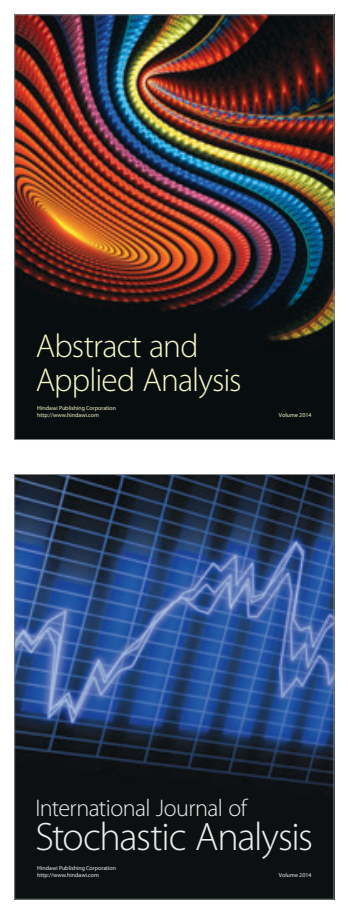

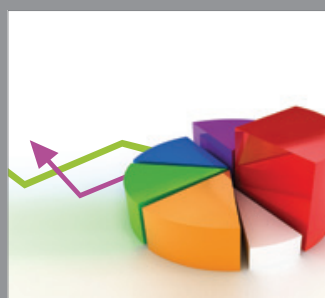

ournal of

Probability and Statistics

Promensencen
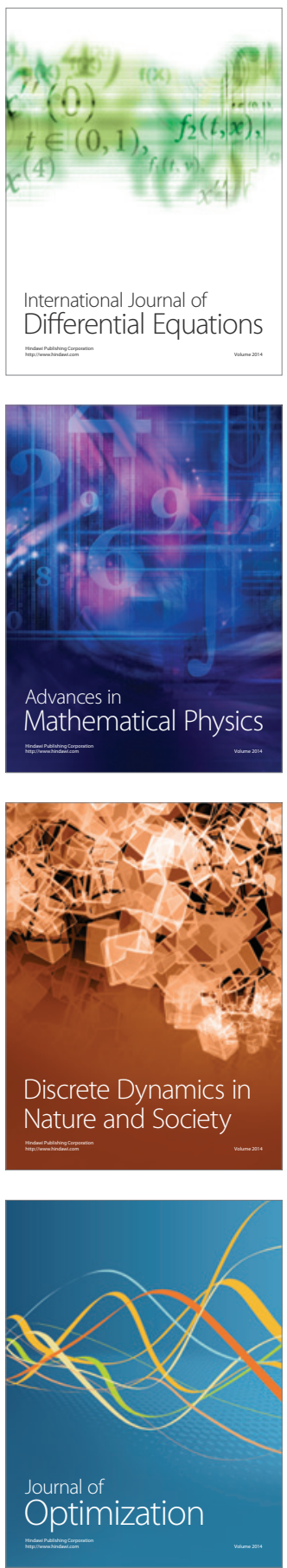